\title{
Finetuning a single-blade sonic paper-cutter as an energy-saving sharpening instrument
}

\author{
Kudzai L. Shava ${ }^{1}$ \\ Carolline Hlakudi ${ }^{1}$ \\ ${ }^{1}$ School of Electrical and Information Engineering \\ University of the Witwatersrand, Johannesburg \\ 1 Jan Smuts Laan \\ Braamfontein 2050 \\ South Africa
}

\author{
Michiel Postema ${ }^{1,2}$ \\ Craig S. Carlson ${ }^{1,2, *}$ \\ ${ }^{2}$ BioMediTech \\ Faculty of Medicine and Health Technology \\ Tampere University \\ Korkeakoulunkatu 3 \\ 33720 Tampere \\ Finland
}

\begin{abstract}
Constant usage and exposure to harsh environments results in a blade to become blunt. Two matching circuits were developed to improve the energy efficiency of an ultrasonic single-blade paper-cutting tool, whilst enhancing the sharpness of the blade. The unmatched ultrasonic knife had a measured magnitude of $-0.3 \mathrm{~dB}$ and a resonance frequency of $251 \mathrm{kHz}$. The incorporation of a matching circuit significantly improved the magnitude indicating greater power transfer and consequently energy efficiency. The sharpening effect of ultrasound was qualitatively and quantitatively investigated, where both yielded a less tortuous cut under sonication in comparison to the unsonicated case. The addition of a matching circuit further improved the cutting efficiency. At an operating voltage of $10 \mathrm{~V}_{\text {peak }}$, the cut made by the ultrasonic knife with a matching circuit was less tortuous than even an industrially cut edge, with a linearity of $61-63 \%$ compared to $59 \%$, respectively.
\end{abstract}

Index Terms-B\#, impedance matching circuit, sharp sonic cutting, tortuosity measurements, ultrasonic knife

\section{INTRODUCTION}

Ultrasound has become useful for a variety of purposes over the years. Innovation has brought about its use in imaging, surgery, cleaning, cell manipulation, food processing, amongst other applications [1]-[5]. An interesting application is the sharpening effect that ultrasound demonstrates on cutting devices by creating microrotations of the blade [6] or by applying the ultrasound vibration directly to a fixed blade [7]. This is particularly of interest since the cutting force could be reduced and the surfaces of the cut would be of higher quality [6]-[9]. Cutting blades inherently become blunt as a result of exposure to harsh environments and repeated use, so

This work has been based on research supported in part by the National Research Foundation of South Africa, Grant Number 127102, and by the Academy of Finland, Grant Number 340026.

*Correspondence to: Craig.Carlson@wits.ac.za a mechanism to sharpen them would improve the efficiency and longevity of the tool.

In this study we fine tuned a single-blade paper-cutting tool by improving the energy efficiency through means of impedance matching. The device in question, an ultrasonic knife shown in Figure 1, comprises a stainless steel surgical scalpel blade attached to a Perspex waveguide supplied with ultrasound through a lead zirconate titanate (PZT) piezoelectric element (Pz37) that is backed using an epoxy resin substrate and enclosed within a perspex enclosure. Existing ultrasonic knives are small scale devices that make use of a generator, booster, ultrasound transducer and blade that are all enclosed into one device removing the hassle of matching impedances [10].

The impedance matching was achieved by developing two Inductor-Capacitor (LC) matching circuit topologies which best matched the electrical impedance of the ultrasonic knife to the impedance of the power amplifier powering it. This

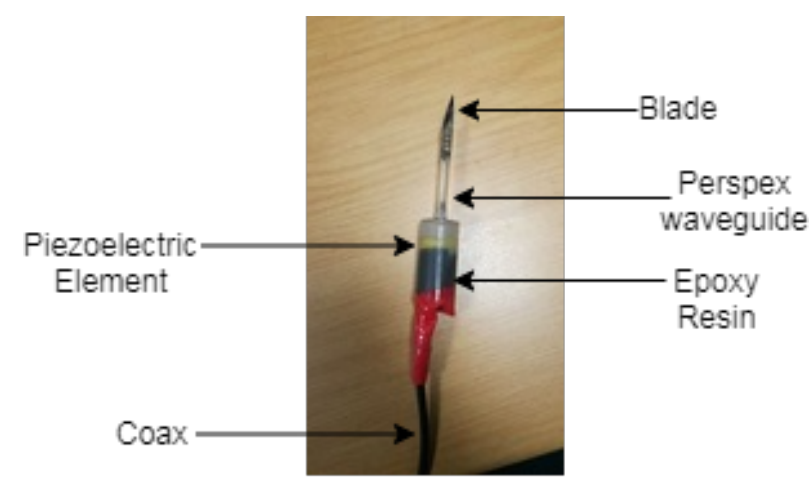

Fig. 1. Ultrasonic knife with a piezoelectric element and a blunted scalpel blade attached with screws to a Perspex waveguide. 
allowed the maximum transfer of power from the amplifier to the knife, thus improving the energy efficiency and cutting ability of the knife. It is important to note that the blade utilised with the device was blunt through repeated use and exposure to the atmosphere. This allowed for us to show the sharpening effect of ultrasound.

\section{BACKGROUND}

\section{A. Ultrasound transducers}

The ultrasound transducer in this study is a transmitter converting electrical signals into ultrasound that is used to vibrate the knife. When a sinusoidal signal is supplied to the transducer, the piezoelectric crystals oscillate at the same frequency and produces sound waves of that frequency. A good transducer maximizes acoustic output whilst having the desired response and bandwidth [11]. The acoustic output has an effect on the efficiency of cutting. However, the transducer used in this study is acoustically matched and thus the study focusses on the electrical matching.

\section{B. Piezoelectric effect}

The piezoelectric effect is when deformation is applied to a material such that negative charges move relative to the positive charges and a dipole moment is developed [11]. This is the principle that the piezoelectric transducer uses in producing sound waves whose vibrations are used in ultrasonic cutting.

\section{Transmission line impedance matching}

Power transferred between a source and a load is greatly reduced by two factors: reflections and power dissipation by a lossy transmission line [12]. A mismatch in the impedance of the load and the source results in reflections. In high power applications, these reflections may damage the source if the reflected power is too high [13]-[15]. The aim is to match the source to the load such that the coefficient of reflection is minimised and maximum power is transferred. The maximum power transfer occurs when the impedances of the load and the source are complex conjugates of each other.

\section{MAterials AND Methods}

\section{A. Experimental setup}

As illustrated in Figure 2, an AFG 3021B arbitrary waveform generator (Tektronix Incorporated, Beaverton, Oregon, United States) was used to generate a continuous signal at the resonance frequency with either a $5 \mathrm{~V}_{\text {peak }}$ or a $10 \mathrm{~V}_{\text {peak }}$ operating voltage, which was fed into an A-150 55-dB linear power amplifier (ENI Technology, Inc., Rochester, New York, United States). This signal was converted to ultrasound using the ultrasonic knife described by [7], coupled with matching circuits. The ultrasonic knife was clamped on a retort stand

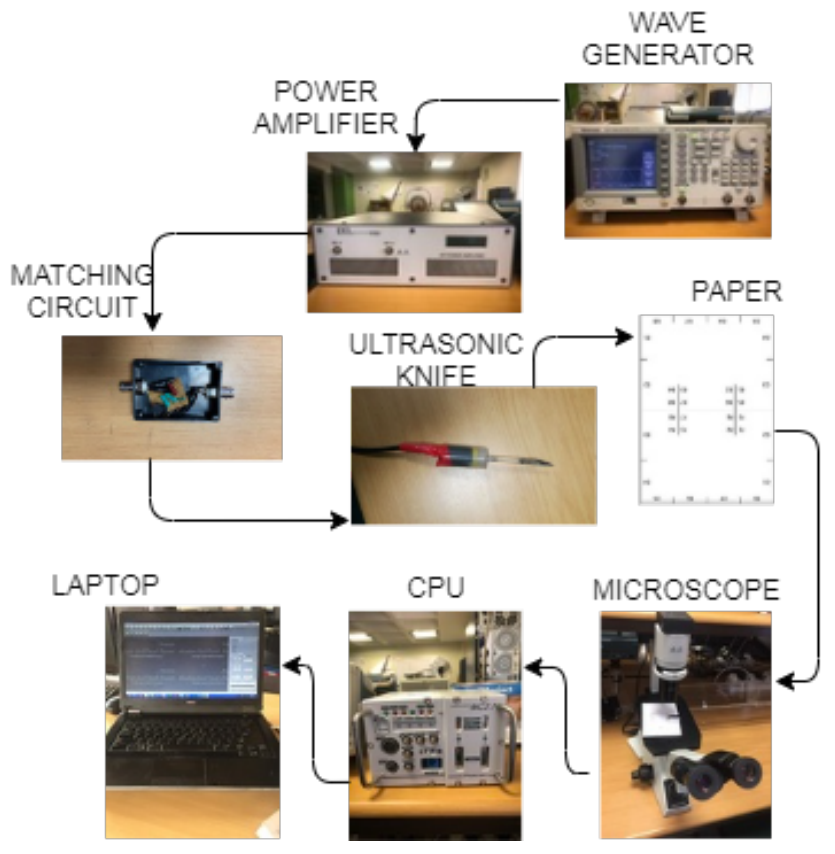

Fig. 2. Flow diagram of the experimental setup used.

and was kept in the same fixed position throughout the experiments.

It is important to match the impedance of the source and load to maximise the power transfer from the source to the load, thus increasing the energy efficiency of the device. This was achieved by developing two matching circuits, each using a different topology as shown in Figure 3. The resonance frequency and magnitude of reflected power relative to the input power were defined as the performance variables. A frequency sweep from $150 \mathrm{kHz}$ to $350 \mathrm{kHz}$ was performed on a ZND Vector Network Analyzer (Rohde \& Schwarz GmbH \& Co. KG, Munich, Germany) with only the ultrasonic knife connected to it, and with each of the matching circuits included to understand the performance variables.

The values of components for the $\mathrm{N}$-type matching circuit were $\mathrm{C} 1=12 \mathrm{nF}, \mathrm{C} 2=3.5 \mathrm{nF}$, and $\mathrm{L} 1=111 \mu \mathrm{H}$. For the T-type matching circuit, the values of the components were $\mathrm{L} 1=548 \mu \mathrm{H}, \mathrm{L} 2=165 \mu \mathrm{H}$, and $\mathrm{C} 1=2.85 \mathrm{nF}$.

Another important consideration is the effect of temperature on the device. An increase in temperature would result in an increase in the resonance frequency of the piezoelectric element [16]. The two input channels of a FLUKE 52II THERMOMETER (Fluke Corporation, Everett, Washington, United States) were physically connected to a position on the blade and to a position to the side of the piezoelectric element. Temperature measurements were taken over a three minute period.

For sharpness testing, plain printer paper was used. Mondi Rotatrim 160C1E ENVIROWHITE $80 \mathrm{~g} / \mathrm{m}^{2}$ A4 paper (Mondi Limited, Melrose Arch, South Africa) was guillotined into 


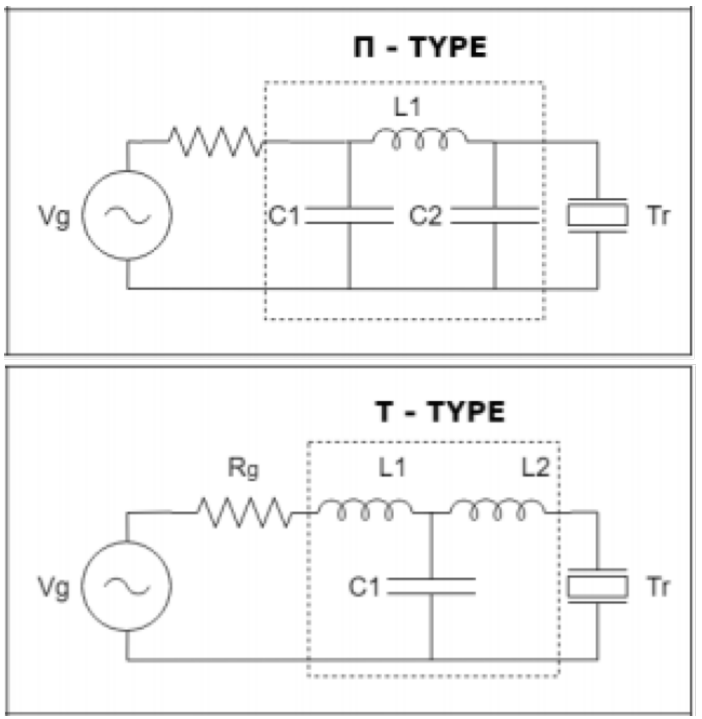

Fig. 3. The N-type and T-type impedance matching circuit topologies used in this study, where $V_{g}$ represents the power amplifier and $T_{r}$ represents the ultrasound transducer.

quarters (equivalent to an A6 page size), and each quarter folded in half. The experimental sheet and cutting procedure used was the same as [7]. Eight experimental sheets were used for each of the unsonicated, sonicated and matching circuit experiments.

The cuts were optically evaluated using a CKX31 inverted microscope (Olympus Corporation, Shinjuku, Tokyo, Japan) with an Olympus C-Plan $10 \times$ (N.A 0.25) objective lens at eight positions on the industrially cut edge and at eight positions on the cuts made.

\section{B. Tortuosity measurement algorithm}

An algorithm to quantify the efficiency of the cuts made was developed using MATLAB ${ }^{\circledR}$ (The MathWorks, Inc., Natick, MA, USA). The algorithm calculated how straight/linear the length of the cut was based on the skeleton boundary extract from the binary image of the cut. Figure 4 shows the flow diagram of the algorithm.

The algorithm read in the original image obtained from the microscope and converted it to gray-scale. The threshold level of the gray-scale image was determined. This was used to binarize the image. If this level of gray threshold was not used, some holes would have remained in the image negatively affecting the results. These holes were filled in by pixels similar to the surrounding pixels. Then the length along the cut was extracted to define the boundary of the image.

The skeleton of the image is the most important property of the image as many applications can be performed on the skeleton [17]. Before the skeleton could be extracted, a dilation operation was performed on the image to increase the width of the boundary. Without this operation, the skeleton image

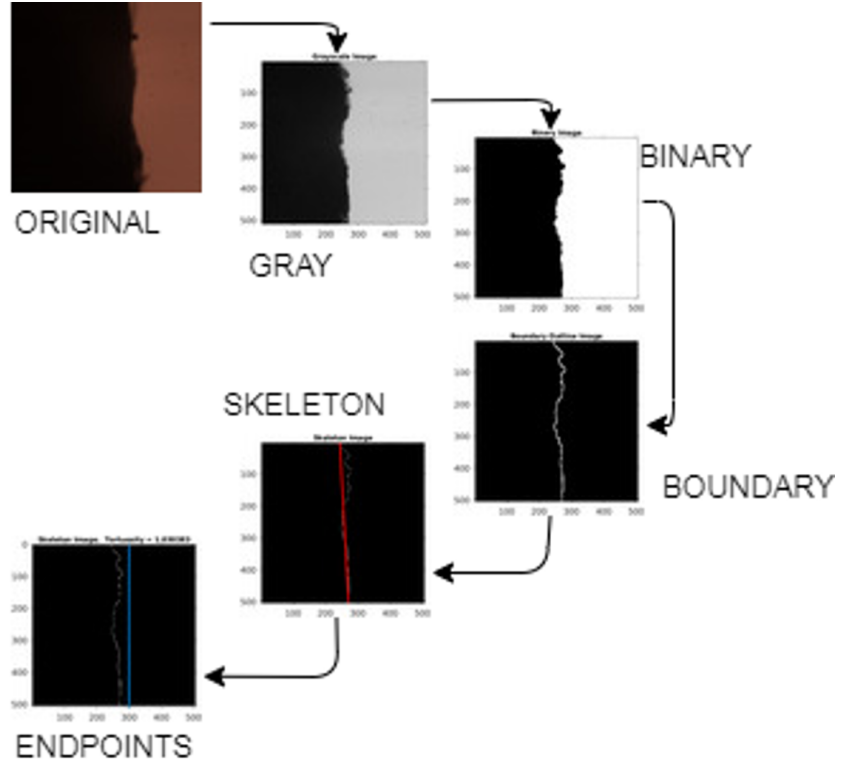

Fig. 4. Flow diagram of the tortuosity measurement algorithm.

would be a discontinuous length that cannot be used to find the length of the skewed cut edge. This is a problem that results from the resolution or instability of the camera, or poor lighting [18]. Once the skeleton image was extracted its endpoints were found, following which the straight-line distance between the marked endpoints was calculated. Finally the tortuosity of the cut, $T$, could be calculated using

$$
T=\frac{L_{\mathrm{B}}}{L_{\mathrm{E}}},
$$

where $L_{\mathrm{B}}$ was the total length of the boundary along the skewed cut edge, and $L_{\mathrm{E}}$ was the straight-line length between the marked endpoints.

\section{Results And Discussion}

At a resonance frequency of $251 \mathrm{kHz}$, the impedance of the ultrasonic knife was found to be $618-j 1195 \mathrm{k} \Omega$ with a magnitude of $-0.3 \mathrm{~dB}$, as shown in Table I. When considering the effect of the matching circuits, there was a significant improvement in the magnitude. The N-type matching circuit has a higher change in the magnitude than the T-type matching circuit. Since the matching circuits yield an increase in the amount of power transferred by the knife, shown by the lower magnitude, it can be concluded that they improve the energy efficiency of the ultrasonic knife.

The matching circuits also shift the resonance frequency of the ultrasonic knife. The shift in resonance frequency may arise due to the frequency dependent nature of the inductors and capacitors used in the matching circuits. To obtain the optimal performance from the ultrasonic knife it should be operated at the resonance frequency. 
TABLE I

EFFECT OF THE MATCHING CIRCUITS ON THE MAGNITUDE AND RESONANCE FREQUENCY OF THE ULTRASONIC KNIFE.

\begin{tabular}{|c|cc|}
\hline Matching Circuit & Magnitude (dB) & Frequency (kHz) \\
\hline N-type & -17 & 294 \\
\hline T-type & -7 & 289 \\
\hline No matching circuit & -0.3 & 251 \\
\hline
\end{tabular}

Table II shows the temperature at two positions after $3 \mathrm{~min}$ utes of sonication. The measurements were conducted at a room temperature of $21^{\circ} \mathrm{C}$.

Whether with or without a matching circuit, the temperature at the blade remains almost unchanged. In comparison, the temperature over the piezoelectric element increases a greater amount with the matching circuit. However, the increase in temperature is still within a safe margin of operation of the piezoelectric element.

Each experimental sheet was optically evaluated along the cuts. This resulted in 64 images for each of the experimental data sets. A representative sample of the industrially cut edge and experimental cuts is shown in Figure 5. Additionally, examples of the effects of the different matching circuit topologies and operating voltages on the cuts are shown in Figure 6.

Visually, the cuts without sonication are significantly more tortuous than any of the other experimental data sets. The cuts resulting from sonication without a matching circuit are less tortuous than the cuts without sonication, but it appears more tortuous in comparison to the industrial edge. Interestingly, the cuts resulting from sonication with a matching circuit appear to be the least tortuous of the datasets. It also appears that the $\mathrm{N}$-type matching circuit at an operating voltage of $10 \mathrm{~V}_{\text {peak }}$ yields the least tortuous cut.

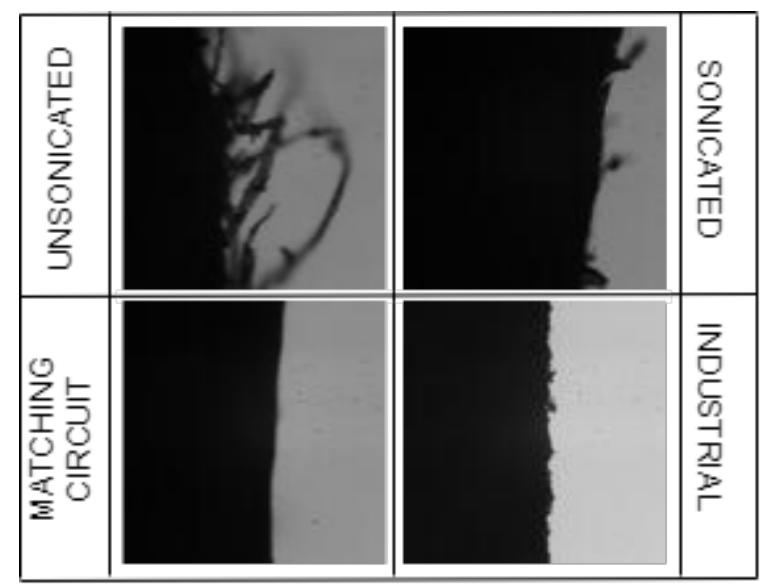

Fig. 5. Back-lit bright-field $10 \times$ microscopy images of cuts in paper from an unsonicated blunt blade, a sonicated unmatched blunt blade, and a sonicated matched blunt blade compared to the paper edge from sharp industrial cutting. The darks areas represent the paper. Each frame corresponds to a $416 \times 416\left(\mu \mathrm{m}^{2}\right)$ area.
TABLE II

TEMPERATURE OF THE SINGLE-BLADE AND PIEZOELECTRIC ELEMENT OF THE ULTRASONIC KNIFE AFTER 3 MINUTES OF USE.

\begin{tabular}{|c|cc|}
\hline & \multicolumn{2}{|c|}{ Temperature $\left({ }^{\circ} \mathbf{C}\right)$} \\
Matching Circuit & Scalpel Blade & Piezoelectric Element \\
\hline N-type & 21.6 & 23.6 \\
\hline T-type & 21.4 & 23.0 \\
\hline No matching circuit & 21.2 & 22.4 \\
\hline
\end{tabular}

To validate the qualitative analysis, a quantitative analysis using the tortuosity algorithm was performed. Table III shows the calculated tortuosities. The value of tortuosity is an average of the tortuosities of the 64 images taken for each experimental dataset.

In should be noted that the ideal straight line is set as the benchmark for calculating linearity. For instance, to calculate the linearity achieved by the N-type matching circuit at $10 \mathrm{~V}_{\text {peak }}$, equation 2 is followed:

$$
L_{\%}=\frac{1}{T} * 100=\frac{1}{1.58} * 100=63.3 \%,
$$

where $L_{\%}$ is the linearity as a percentage.

Both qualitative and quantitative analysis indicate that sonication of a single-blade paper-cutting tool will improve the efficiency of the cuts. Introducing matching circuits will improve the power transfer to the tool, which in turn results in even further improved efficiency of the cuts. Additionally, to improve the efficiency of cutting of an originally blunt blade to exceed that of an industrial cutting machine, the higher operating voltage is required. It can be clearly seen how the sonicated $\mathrm{N}$-type matching circuit dataset operating at a voltage of $10 \mathrm{~V}_{\text {peak }}$ has the highest linearity of $61.3 \%$, while the industrially cut edge only has a linearity of $58.8 \%$.

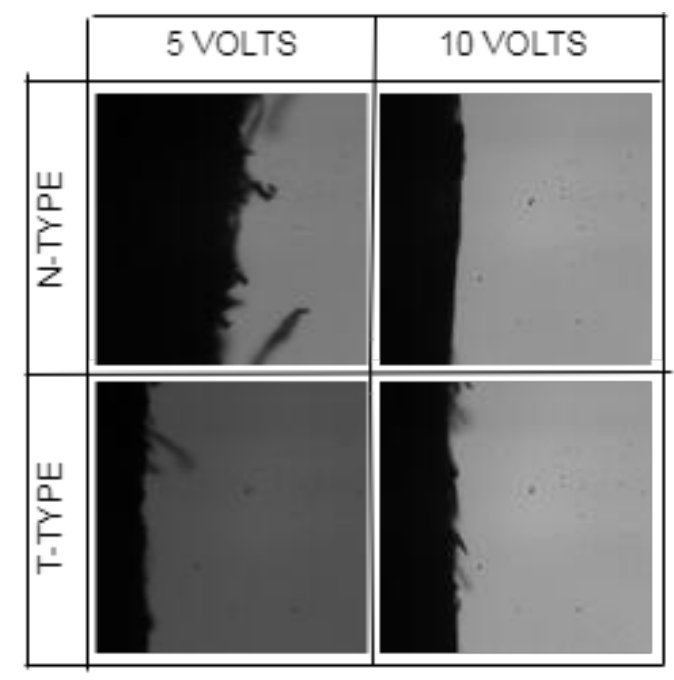

Fig. 6. Back-lit bright-field $10 \times$ microscopy images of cuts in paper made at two different voltages with two different matching circuits. The darks areas represent the paper. Each frame corresponds to a $416 \times 416\left(\mu \mathrm{m}^{2}\right)$ area. 
TABLE III

TORTUOSITIES OBTAINED UNDER DIFFERENT CONDITIONS.

\begin{tabular}{|c|cc|cc|}
\hline & \multicolumn{3}{|c|}{ Tortuosity } & \multicolumn{2}{c|}{ Linearity (\%) } \\
Experimental Dataset & $5 V_{p}$ & $10 V_{p}$ & $5 V_{p}$ & $10 V_{p}$ \\
\hline N-type matching & 1.85 & 1.58 & 54.1 & 63.3 \\
\hline T-type matching & 1.71 & 1.63 & 58.5 & 61.3 \\
\hline Sonicated & 1.89 & 1.87 & 52.9 & 53.5 \\
\hline Unsonicated & \multicolumn{2}{|c|}{1.90} & \multicolumn{2}{|c|}{52.6} \\
\hline Industrial & \multicolumn{2}{|c|}{1.70} & \multicolumn{2}{c|}{58.8} \\
\hline
\end{tabular}

\section{CONCLUSiON}

Energy efficient power transfer and improved cutting ability of an ultrasonic knife was the focus of this study. Two matching circuits were developed to match the impedance of the ultrasonic knife and the power amplifier to maximise power transfer. The sharpening effect of ultrasound and the incorporating of matching circuits was qualitatively and quantitatively investigated.

The ultrasonic knife had a resonance frequency of $251 \mathrm{kHz}$ and a measured magnitude of $-0.3 \mathrm{~dB}$. When incorporating the matching circuits, there was a significant increase in the magnitude indicating a greater transfer of power and thus improved efficiency of energy usage. An interesting observation was the shift of the resonance frequency when the matching circuits were incorporated.

The cuts resulting from sonication were visibly less tortuous than the cuts without sonication, and had as many or fewer visible paper fibres when compared to an industrially cut edge. The quantitative analysis supports these qualitative observations, where a higher operating voltage yielded a lower tortuosity. The percentage linearity of the ultrasonic knife with the N-type matching circuit operating at $294 \mathrm{kHz}$ and an input voltage of $10 \mathrm{~V}_{\text {peak }}$ was $63.3 \%$ whilst the unsonicated cut and industrially cut edge was $52.6 \%$ and $58.8 \%$ respectively. Therefore, it can be concluded that utilising a matching circuit and ultrasound to fine tune a single-blade paper-cutting tool enhances the overall energy and cutting efficiency.

\section{REFERENCES}

[1] P. N. T. Wells, "Ultrasound imaging," Physics in Medicine and Biology, vol.51, no. 13, pp. R83-R98, 2016. [DOI: 10.1088/0031-
9155/51/13/R06]

[2] A. R. Khan, M. Hafeez, and A. U. Arif: "Ultrasonic cutting in multinodal thyroid swellings", Journal of Medical Sciences, vol. 27, no. 1, pp. 7-10, 2019.

[3] T. J. Mason, "Ultrasonic cleaning: An historical perspective," Ultrasonics Sonochemistry vol.29, pp.519-523, 2016. [DOI: 10.1016/j.ultsonch.2015.05.004]

[4] D. M. Rubin, N. Anderton, C. Smalberger, J. Polliack, M. Nathan, and M. Postema, "On the behaviour of living cells under the influence of ultrasound," Fluids, vol. 3, no. 4, 82, 2018. [DOI: 10.3390/fluids3040082]

[5] N. Bhargava, R. S. Mor, K. Kumar, and V. S. Sharanagat, "Advances in application of ultrasound in food processing: a review," Ultrasonics Sonochemistry, vol.70, 105293, 2021. [DOI: 10.1016/j.ultsonch.2020.105293]

[6] G. Sinn, B. Zettl, H. Mayer, and S. Stanzl-Tschegg: "Ultrasonic-assisted cutting of wood", Journal of Materials Processing Technology, vol. 170, no. 1-2, pp. 42-49, 2005. [DOI: 10.1016/j.jmatprotec.2005.04.076]

[7] C. S. Carlson, A. Pohl, D. G. Keir, and M. Postema, "Cutting edge technology: sound sharpens the blade," Applied Acoustics, vol. 166, 107336, 2020. [DOI: 10.1016/j.apacoust.2020.107336]

[8] G. A. Arnold, S. Zahn, A. Legler, and H. Rohm, "Ultrasonic cutting of foods with inclined moving blades," Journal of Food Engineering, vol.103, no. 4, pp.394-400, 2011. [DOI: 10.1016/j.jfoodeng.2010.11.009]

[9] J. Wang, F. Fang, X. Zhang, and D. De Simone, "Investigation on controlling diamond tool edge and wear by ultrasonic vibration," Applied Acoustics, vol. 176, 107896, 2021. [DOI: 10.1016/j.apacoust.2020.107896]

[10] L. L. Hood, "Improved ultrasonic knife," Journal of the Acoustical Society of America, vol. 95, no. 4, pp. 2296, 1994. [DOI: 10.1121/1.408656]

[11] M. Postema, Fundamentals of Medical Ultrasonics. Taylor \& Francis: London, 2011. [DOI: 10.1201/9781482266641]

[12] R. J. Vernon, and S. R. Seshadri, "Reflection coefficient and reflected power on a lossy transmission line," Proceedings of the IEEE, vol. 57, no. 1, pp. 101-102, 1969. [DOI:10.1109/PROC.1969.6893]

[13] H. Zhou, S. H. Huang, and W. Lei, "Electrical impedance matching between piezoelectric transducer and power amplifier," IEEE Sensors Journal, vol.20, no.23, pp.14273-14281, 2021. [DOI: 10.1109/JSEN.2020.3008762]

[14] V. T. Rathod, "A review of electric impedance matching techniques for piezoelectric sensors, actuators and transducers," Electronics vol. 8 no. 2, 169, 2019. [DOI: 10.3390/electronics8020169]

[15] B. A. Auld, Acoustic Fields and Waves in Solids, $2^{\text {nd }}$ ed. Krieger Publishing Company: Florida, 1990.

[16] V. Upadhye, and S. Agashe, "Effect of temperature and pressure variations on the resonant frequency of piezoelectric material," Measurement and Control, vol.49, no. 9, pp.286-292, 2016. [DOI: 10.1177/0020294016663974]

[17] M. Bao, S. Guo, Q. Tang, and F. Zhang, "Optimization of the bwmorph function in the MATLAB image processing toolbox for binary skeleton computation," 2009 International Conference on Computational Intelligence and Natural Computing, pp.273-276, 2009. [DOI: 10.1109/CINC.2009.96]

[18] H. N. Ghafil and D. M. B. Ali, "Cracks measurement on the basis of machine vision," International Journal of Video \& Image Processing and Network Security, vol. 16, no. 6, pp. 1-6, 2016. 